\title{
Effect of Freeze-Drying Conditions on Antioxidant Compounds of Broccoli
}

\author{
Andrea Mahn*, Mauricio Zamorano and Alejandro Reyes
}

Department of Chemical Engineering, Universidad de Santiago de Chile, Santiago, Chile

\begin{abstract}
The effect freeze-drying conditions, i.e. particle size, use of infrared radiation, and drying air temperature in Atmospheric Freeze-Drying (AFD) or freezing rate in vacuum Freeze-Drying (VFD) on the total selenium content, total polyphenols content and anti-radical power of broccoli was investigated. Two factorial designs were used, with three factors in two levels each one, for each type of freeze-drying. In AFD, the experimental factors were drying air temperature $\left(5^{\circ} \mathrm{C}\right.$ or $\left.15^{\circ} \mathrm{C}\right)$, particle size $(1.0$ or 1.5 $\mathrm{cm}$ equivalent diameter), and use of infrared radiation (IR; yes or no). In VFD, the factors were freezing rate (high: by immersion in liquid nitrogen, low: in a household freezer at $-30^{\circ} \mathrm{C}$ during $24 \mathrm{~h}$ ), particle size and IR use, in the same levels as for AFD. In AFD, all experimental factors significantly affected the antioxidant properties of broccoli, while in VFD only the total polyphenols content was affected by freezing rate. Both AFD and VFD impaired antioxidant properties of the processed vegetable to different extents; however the content of total polyphenols and Se were considerably higher in broccoli subjected to AFD. Then AFD is an attractive alternative for broccoli preservation since it allows keeping some healthy properties of the vegetable. Finally, this technology seems promising in the functional food industry considering broccoli as raw material for this use.
\end{abstract}

\section{Keywords: Broccoli; Freeze-drying; Antioxidants}

\section{Introduction}

Broccoli exhibits high content of antioxidants, mainly polyphenols and minerals such as selenium [1]. Selenium is a key trace element that acts as cellular antioxidant, thus improving the immune system and reducing the risk of developing cardiovascular diseases and some types of cancer [2]. Broccoli has the capability of accumulating Se, fact closely linked to cancer prevention [3]. On the other hand, polyphenols are known to reduce the risk of developing degenerative diseases triggered by oxidative stress [4]. Processing usually impairs the nutritional quality of vegetables. Since broccoli is mostly consumed processed, elucidating the processing conditions that minimize nutritional losses becomes relevant. The effect of operating conditions in dehydration processes, other than convective drying, on the antioxidant compounds of broccoli has been poorly studied [5]. Recently, Mahn et al. [6] studied the effect of freeze-drying conditions on the content of polyphenols, organic selenium and antioxidant capacity of selenium - enriched broccoli previously subjected to an optimized blanching step. They found that low freezing rate; application of infrared radiation, and atmospheric pressure maximized the content of organic-selenium in the final product. However, antioxidant capacity and total polyphenols content dramatically decreased in comparison with the fresh vegetable.

In this work we extend the results of Mahn et al. [6] to traditionally cultured broccoli, by analyzing both atmospheric freeze-drying and vacuum freeze-drying independently. The effect of particle size, use of infrared radiation, and drying air temperature or freezing rate in atmospheric freeze-drying (AFD) and Vacuum Freeze-Drying (VFD) on the content of total selenium, total polyphenols and antioxidant capacity of broccoli was investigated.

\section{Materials and Methods}

\section{Experimental design}

Two factorial designs were used, with three factors in two levels each one, for each type of freeze-drying: atmospheric Freeze-Drying (AFD) and vacuum Freeze-Drying (VFD). In AFD, the experimental factors were drying air temperature $\left(5^{\circ} \mathrm{C}\right.$ or $\left.15^{\circ} \mathrm{C}\right)$, particle size $(1.0$ or $1.5 \mathrm{~cm}$ equivalent diameter), and use of infrared radiation (IR; yes or no). In VFD, the factors were freezing rate (high: by immersion in liquid nitrogen, low: in a household freezer at $-30^{\circ} \mathrm{C}$ during $24 \mathrm{~h}$ ), particle size and IR use, in the same levels as for AFD. The experimental runs were executed in a random order. Measurements were performed in duplicate. Table 1 shows the experimental matrix in standard order.

\section{Sample preparation}

Broccoli florets were purchased at the local market. First, florets were cut in $1 \mathrm{~cm}$ or $1.5 \mathrm{~cm}$ equivalent diameter particles. Since florets are not exactly spherical, particle volume was measured as the displaced water volume, and it was assumed that this volume corresponded to a sphere, and then the equivalent diameter was calculated [7]. After that, broccoli particles were subjected to a short hydrothermal treatment consisting of immersing broccoli particles in a $90^{\circ} \mathrm{C}$ water bath during 2 min and immediately after cooled in an ice bath. Excess water was withdrawn from broccoli particles prior drying. Approximately $100 \mathrm{~g}$ broccoli florets were dried in each experimental run.

\section{Freeze-drying}

The VFD runs were carried out in a commercial Labconco (Kansas, MI, USA) freeze-dryer, implemented with a $150 \mathrm{~W}$ infrared light bulb beside the flask containing the product, in order to ensure that most of the IR light hit onto the particles. The temperature of the condenser was set to $-48^{\circ} \mathrm{C}$ and the inner pressure of the chamber was $60 \mathrm{~Pa}$. The AFD runs were carried out in a vertical drying chamber where dry air circulates after passing through a silica gel bed. To ensure the dryness of the drying air, the silica gel was replaced every 2 hours. The system was provided with an IR source (Tungsram, $150 \mathrm{~W}$, Infrarubin, Hungary). Both equipments were operated as described by Reyes et al. [8].

*Corresponding author: Andrea Mahn, Department of Chemical Engineering Universidad de Santiago de Chile, Santiago, Chile, Tel: +56 7181833; E-mail: andrea.mahn@usach.cl

Received July 16, 2014; Accepted August 31, 2014; Published September 04 2014

Citation: Mahn A, Zamorano M, Reyes A (2014) Effect of Freeze-Drying Conditions on Antioxidant Compounds of Broccoli. J Food Process Technol 5: 360. doi:10.4172/2157-7110.1000360

Copyright: () 2014 Mahn A, et al. This is an open-access article distributed under the terms of the Creative Commons Attribution License, which permits unrestricted use, distribution, and reproduction in any medium, provided the original author and source are credited. 


\begin{tabular}{|c|c|c|c|c|c|c|}
\hline \multicolumn{7}{|c|}{ Atmospheric freeze-drying } \\
\hline Run & $\mathrm{T}\left({ }^{\circ} \mathrm{C}\right)$ & Particle size $(\mathrm{cm})$ & Use of IR & ARP (mL/mg) & Se $(\mu g / K g D M)$ & TPP (mgGAE/gDM) \\
\hline $1 \mathrm{~A}$ & 5 & 1.0 & No & $0.996 \pm 0.008$ & $489 \pm 44$ & $576 \pm 136$ \\
\hline $2 A$ & 15 & 1.0 & No & $0.641 \pm 0.028$ & $83 \pm 12$ & $584 \pm 278$ \\
\hline $3 \mathrm{~A}$ & 5 & 1.5 & No & $0.861 \pm 0.119$ & $427 \pm 43$ & $497 \pm 54$ \\
\hline $4 \mathrm{~A}$ & 15 & 1.5 & No & $0.814 \pm 0.204$ & $66 \pm 12$ & $439 \pm 41$ \\
\hline $5 \mathrm{~A}$ & 5 & 1.0 & Yes & $0.968 \pm 0.182$ & $149 \pm 54$ & $680 \pm 388$ \\
\hline $6 \mathrm{~A}$ & 15 & 1.0 & Yes & $0.470 \pm 0.063$ & $70 \pm 4$ & $785 \pm 208$ \\
\hline $7 \mathrm{~A}$ & 5 & 1.5 & Yes & $0.607 \pm 0.139$ & $73 \pm 53$ & $403 \pm 63$ \\
\hline $8 \mathrm{~A}$ & 15 & 1.5 & Yes & $0.429 \pm 0.224$ & $75 \pm 4$ & $483 \pm 41$ \\
\hline \multicolumn{7}{|c|}{ Vacuum freeze-drying } \\
\hline Run & Freezing rate & Particle size (cm) & Use of IR & ARP (mL/mg) & Se $(\mu \mathrm{g} / \mathrm{KgDM})$ & Polyphenols (mgGAE/gDM) \\
\hline $1 \mathrm{~V}$ & Low & 1.0 & No & $0.961 \pm 0.153$ & 471 & $399 \pm 19$ \\
\hline $2 \mathrm{~V}$ & High & 1.0 & No & $2.493 \pm 0.557$ & 151 & $525 \pm 120$ \\
\hline $3 \mathrm{~V}$ & Low & 1.5 & No & $0.820 \pm 0.023$ & 385 & $384 \pm 1$ \\
\hline $4 \mathrm{~V}$ & High & 1.5 & No & $1.812 \pm 0.730$ & 141 & $530 \pm 25$ \\
\hline $5 \mathrm{~V}$ & Low & 1.0 & Yes & $1.189 \pm 0.040$ & $48 \pm 1$ & $396 \pm 16$ \\
\hline $6 \mathrm{~V}$ & High & 1.0 & Yes & $2.401 \pm 0.127$ & $14 \pm 4$ & $652 \pm 246$ \\
\hline $7 \mathrm{~V}$ & Low & 1.5 & Yes & $0.779 \pm 0.270$ & $32 \pm 5$ & $365 \pm 3$ \\
\hline $8 \mathrm{~V}$ & High & 1.5 & Yes & $2.164 \pm 0.955$ & $21 \pm 4$ & $674 \pm 170$ \\
\hline
\end{tabular}

Table 1: Anti-radical power (ARP), total Se concentration (se) and total polyphenols (TPP) content in broccoli florets subjected to AFD or VFD.

\section{Selenium}

Broccoli samples $(0.2 \mathrm{~g})$ were subjected to acidic digestion by adding acid solution $\left(1.6 \mathrm{~mL} 65 \% \mathrm{HNO}_{3}+0.2 \mathrm{~mL} 70 \% \mathrm{HCLO}_{4}+0.2 \mathrm{~mL}\right.$ $95 \% \mathrm{H}_{2} \mathrm{SO}_{4}$ ), followed by heating at $120^{\circ} \mathrm{C}$ during $3 \mathrm{~h}$ obtaining a final sample volume of $0.3 \mathrm{~mL}$. After that, $\mathrm{Se}(+\mathrm{VI})$ was reduced to $\mathrm{Se}(+\mathrm{IV})$ by adding $1 \mathrm{~mL}$ of $12 \% \mathrm{HCL}$ and heating at $120^{\circ} \mathrm{C}$ for $20 \mathrm{~min}$. Then, $1 \mathrm{~mL}$ of $25 \% \mathrm{NH}_{4} \mathrm{OH}$ was added. Selenium was extracted in an ammonium pyrrolidine dithiocarbamate-methyl isobutylketone (APDC-MIBK) system, and analyzed by atomic absorption spectrophotometry with graphite furnace (Perkin Elmer, Waltham, MA, USA) at $196.1 \mathrm{~nm}$ and $\mathrm{D}_{2}$ background correction [9].

\section{Antioxidants extraction}

The extraction of antioxidant compounds was performed as reported by Vinson et al. [10]. A 200-mg aliquot of fresh or dried broccoli was weighed in a vial and $4 \mathrm{~mL}$ of 80:20 (mL: $\mathrm{mL})$ methanol/water solution was added. The mixture was ultrasound-processed through a titanium probe immersed in the vial for three minutes at $80 \%$ duty cycle Misonix XL ultrasonic liquid-processors 200; Arquimed, NewYork, USA). Then the samples were incubated at room temperature in an orbital shaker for $4 \mathrm{~h}$. After that, samples were centrifuged at 12000xg for $5 \mathrm{~min}$ to remove the solids. The supernatant was recovered and 80:20 (mL:mL) methanol/water solution was added to complete $5 \mathrm{~mL}$ extract volume.

\section{Total polyphenols content}

The total polyphenols content was determined spectrophotometrically through the Folin-Ciocalteau method [10] with some modifications. A $40 \mu \mathrm{L}$ aliquot extract and $200 \mu \mathrm{L}$ FolinCiocalteau reagent were added to $3.16 \mu \mathrm{L}$ distilled water. The mixture was left in darkness at room temperature for $5 \mathrm{~min}$. Then, $600 \mu \mathrm{L}$ of a $20 \%(\mathrm{w} / \mathrm{v})$ sodium carbonate solution was added and left in darkness for another 2 hours. After that, samples were centrifuged at $12000 \mathrm{xg}$ for $5 \mathrm{~min}$ to remove the precipitate, and absorbance was measured at $750 \mathrm{~nm}$ in a Rayleigh UV Bios 1606 spectrophotometer (Rayleigh Instruments Limited, Rayleigh, England). The results were expressed as $\mathrm{mg}$ of gallic acid equivalents (GAE) per liter of extract (mg/L), and then converted to mg GAE per g dry matter. All chemicals were analytical grade and were purchased form Sigma-Aldrich (Schnelldorf, Germany).

\section{Antioxidant capacity}

The antioxidant capacity was determined based on the free radical sequestering ability of the compounds present in the broccoli extracts, using the stable radical 2,2-diphenyl-1-picryhydrazyl (DPPH) [11]. Fifty $\mu \mathrm{L}$ broccoli extract were mixed with $1.950 \mu \mathrm{L}$ DPPH solution (6.5 $\times 10^{-5} \mathrm{M}$ in methanol). The decrease in absorbance at $517 \mathrm{~nm}$ was recorded until reaching a plateau. The free radicals inhibition percentage (\%FRI) was determined according to eqn (1), where $\mathrm{A}_{(\mathrm{t}=0}$ ${ }_{\min )}$ is the absorbance of the control mixture (without extract) at zero time, and $\mathrm{A}_{(\mathrm{t}=30 \mathrm{~min})}$ is the absorbance of the reaction mixture (with extract) after 30 minutes reaction.

$$
\% \mathrm{FRI}=\frac{\mathrm{A}_{(\mathrm{t}=0 \mathrm{~min})}-\mathrm{A}_{(\mathrm{t}=30 \mathrm{~min})}}{\mathrm{A}_{(\mathrm{t}=0 \mathrm{~min})}} \times 100
$$

It was determined the antioxidant concentration that reduces to a $50 \%$ the $\mathrm{DPPH}$ concentration in the mixture $\left(\mathrm{EC}_{50}\right)$. A calibration curve was built correlating the polyphenols content with the $\% \mathrm{FRI}$, and $\mathrm{EC}_{50}$ was determined through the regression equation, expressing it as

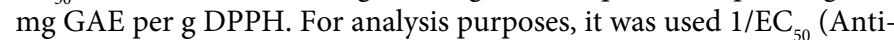
radical power, ARP), as suggested by Brand-Williams et al. [12].

\section{Statistical analysis}

The effects of the experimental factors and their significance (95\% confidence) were determined according to standard procedures [13]. Statistically significant differences in the antioxidant properties of fresh and lyophilized broccoli were determined through Student's t test at 95\% confidence. The software Statgraphics Plus 5.1 (Statistical Graphics Corp., USA, 2000) was used in these analyses.

\section{Results and Discussion}

Table 1 shows the values of Anti-Radical Power (ARP), total selenium concentration, and total polyphenols content. Here it results clear that the type of freeze-drying (atmospheric or vacuum) affected the total selenium concentration, and even further, in all AFD runs this concentration was considerably higher.

Figure 1 shows the standardized effects of drying air temperature, 
particle size, use of infrared radiation, and the binary interactions, on the antioxidant properties of broccoli subjected to AFD. The standardized effects of the experimental factors correspond to the magnitude of the change in the response when the factor moves from the low $(-1)$ to the high level $(+1)$. The results show that the anti-radical power was significantly affected by all the experimental factors as well as by their binary interactions. Air temperature, particle size and use of IR produced a reduction of the anti-radical power. Air temperature and IR application significantly diminished the total Se concentration. Particle size had a significant negative effect on total polyphenols content, i.e. larger particles showed a lower polyphenols content.

Figure 2 shows the standardized effects of freezing rate, particle size, use of infrared radiation, and the binary interactions between the factors, on the antioxidant properties of broccoli subjected to VFD. No factor had significant effect on anti-radical power and total Se concentration. Freezing rate significantly affected total polyphenols content, in a positive way. This means that high freezing rate (by immersion in liquid nitrogen) resulted in higher polyphenols content.

Figure 3 shows a comparison of the average anti-radical power, total Se concentration and total polyphenols content in fresh broccoli and broccoli subjected to AFD or VFD. It is clear that the antioxidant properties of broccoli were affected by the freeze-drying conditions in different ways. The anti-radical power was significantly reduced by freeze-drying, showing a 56.5\% loss in VFD and $80.1 \%$ loss in AFD. Besides, significant differences were detected in this property between VFD and AFD $(\mathrm{p}=0.0064)$. The total polyphenols content was reduced in $48.7 \%$ by VFD and in $41.9 \%$ by AFD. Despite AFD resulted in a higher polyphenols content, no statistically significant differences between VFD and AFD were detected $(\mathrm{p}=0.3173)$. The values obtained in this work for anti-radical power and total polyphenols content are considerably higher than those reported by Mahn et al. for the freeze-drying conditions that maximized selenium content. This differences can be attributed to the blanching treatment, since Mahn et al. optimized this process before freeze-drying, and it consisted of immersion in water at $72^{\circ} \mathrm{C}$ during $1 \mathrm{~min}$, while in this work blanching consisted of immersion in water at $90^{\circ} \mathrm{C}$ during $2 \mathrm{~min}$.

The total Se concentration was significantly higher in AFD ( $\mathrm{p}=$ 0.0298), in comparison with VFD, probably due to a lower volatilization of seleno-compounds in AFD. This result agrees with Mahn et al., who reported that the maximum organic Selenium content in freezedried broccoli was achieved using atmospheric pressure. This is quite interesting, since Se is largely responsible for the anti-carcinogenic properties of broccoli.

Studies about the effect of freeze-drying conditions on the nutritional properties of plant foods have been reported. Reyes et al. [14] investigated the effect of AFD and VFD on nutritional properties of apples. They concluded that AFD produced nutritional losses similar to convective drying. This result agrees with the present study regarding antioxidant properties.

Elucidating the operating conditions that keep Se concentration, anti-radical power and/or total polyphenols content as high as possible in processed broccoli florets is of great usefulness, when thinking about broccoli as raw material for functional foods or an anti-carcinogenic "nutraceutical". It must be considered that broccoli is mostly consumed as a processed food, usually boiled, and hence there is a great loss of antioxidant and anti-carcinogenic compounds that are found in the fresh vegetable. Then, designing alternative processes to extend the shelf life and to minimize nutritional losses is essential to generate a broccoli-based food with higher added value.
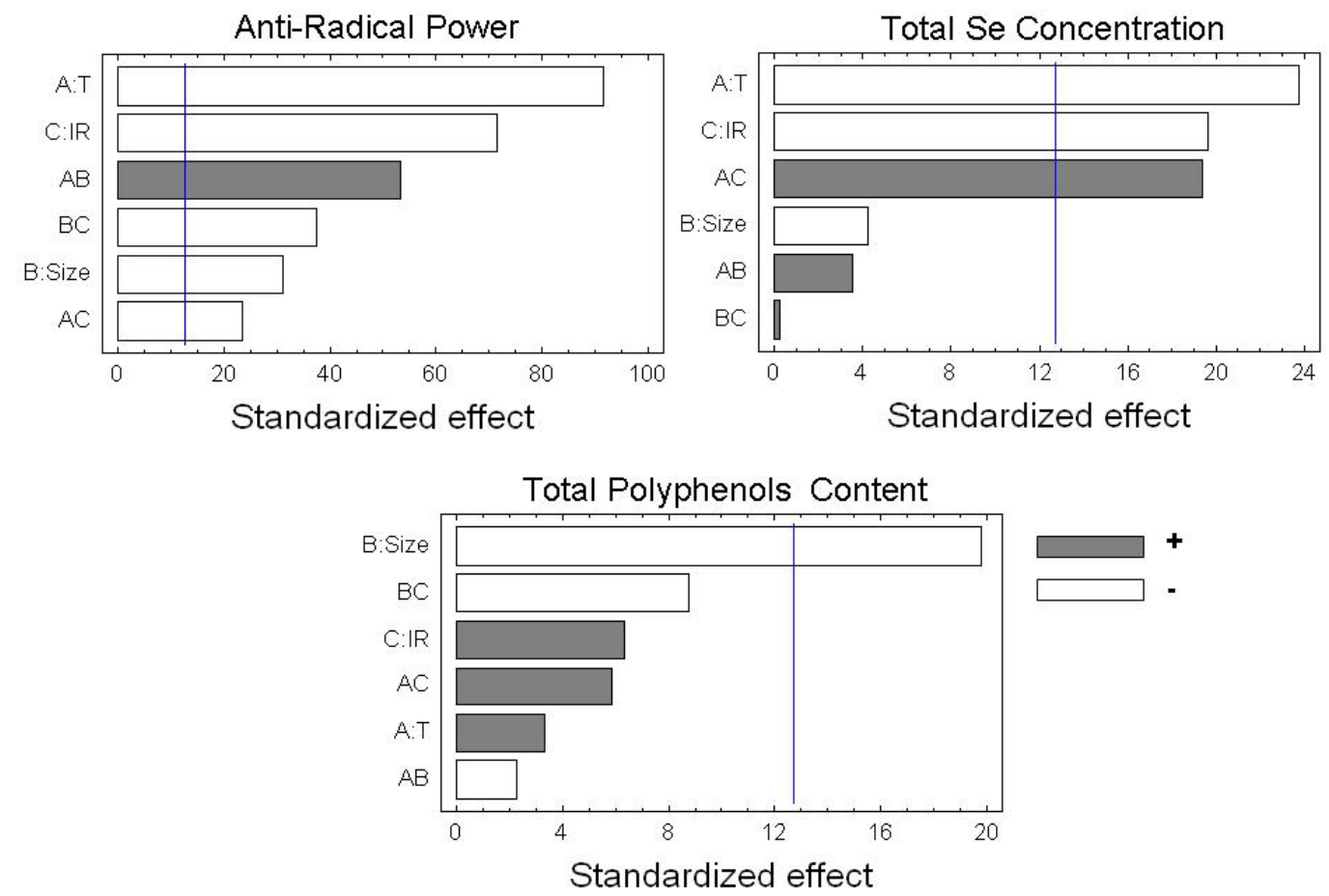

Figure 1: Pareto charts of the standardized effects of the experimental factors in AFD and their interactions on the antioxidant properties of broccoli: anti-radical power, total Se concentration, and total polyphenols content. Filled bars indicate positive effects, empty bars indicate negative effects, FR means freezing rate, and IR means infrared radiation. The bars exceeding the vertical line have a significant effect on the response. 

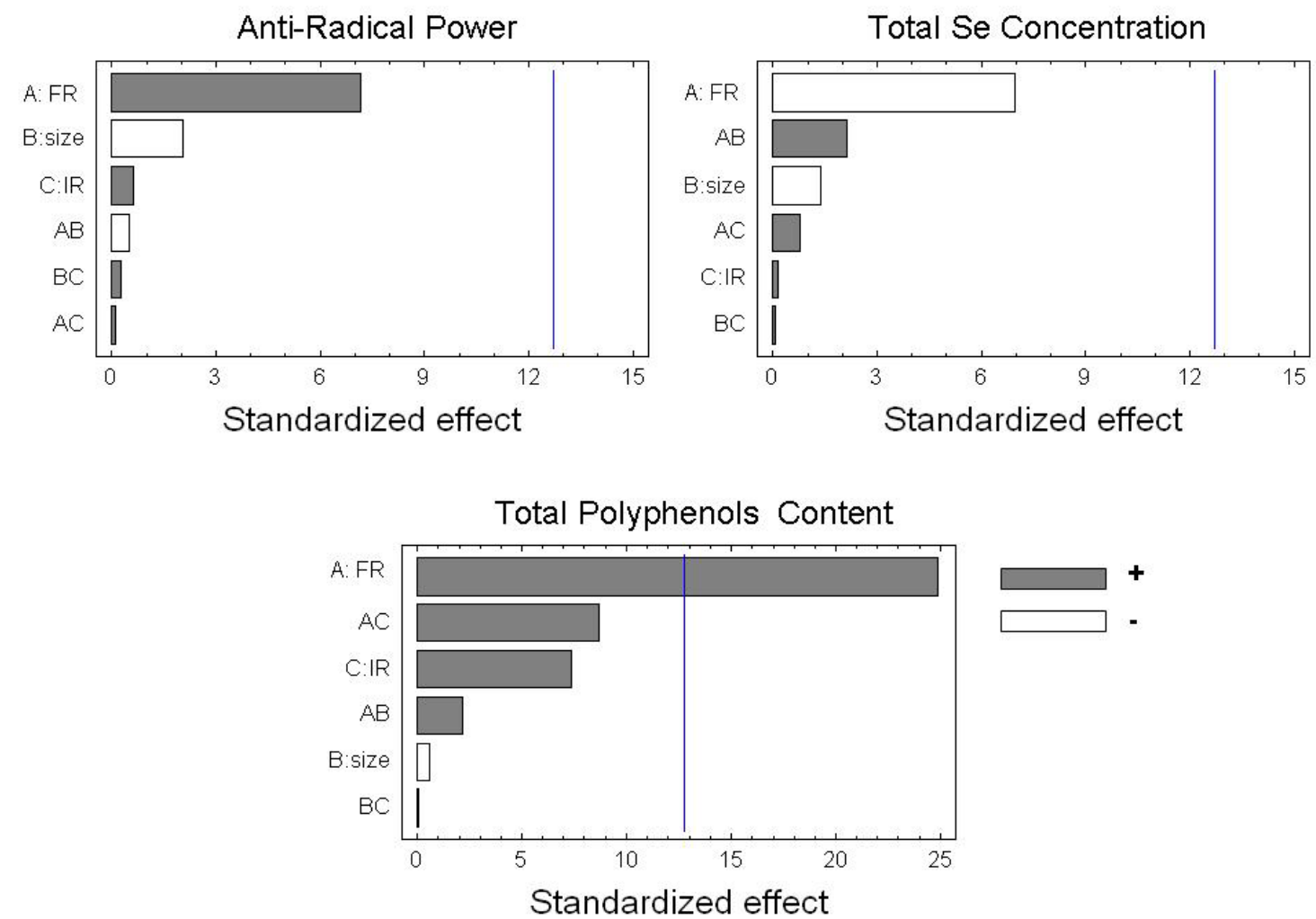

Figure 2: Pareto charts of the Standardized effects of the experimental factors in VFD and their interactions on the antioxidant properties of broccoli: anti-radical power total Se concentration, and total polyphenols content. Filled bars indicate positive effects, empty bars indicate negative effects, FR means freezing rate, and IR means infrared radiation. The bars exceeding the vertical line have a significant effect on the response.

\section{Anti-radical Power}

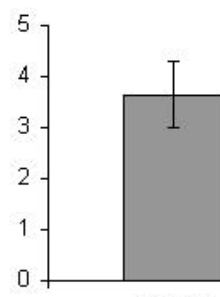

FRESH

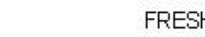

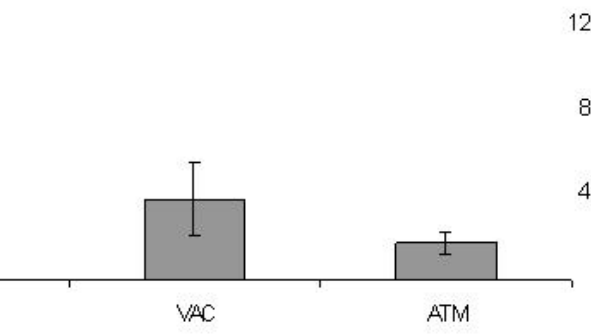

$\mathrm{VAC}$
ATM

\section{Total Polyphenols Content}

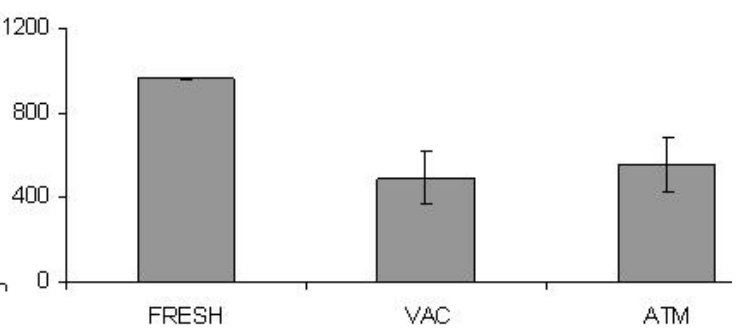

Total Se Concentration

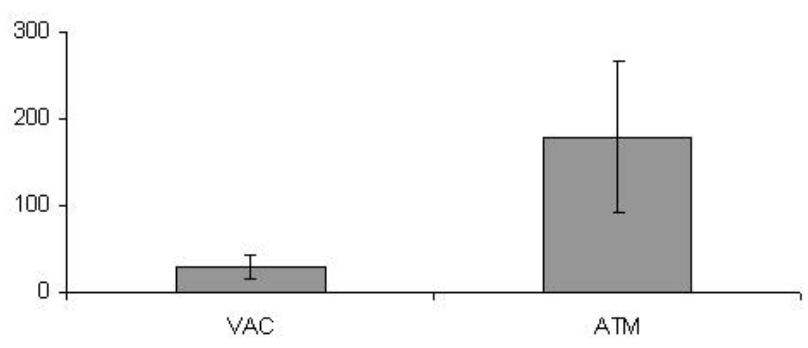

Figure 3: Comparison of the antioxidant properties of fresh, vacuum freeze-dried and atmospheric freeze-dried broccoli. Total Se concentration in fresh broccoli is not reported since the protocol for its determination implies dehydration. 
Citation: Mahn A, Zamorano M, Reyes A (2014) Effect of Freeze-Drying Conditions on Antioxidant Compounds of Broccoli. J Food Process Technol 5: 360. doi:10.4172/2157-7110.1000360

\section{Conclusions}

In summary, both AFD and VFD impair the antioxidant properties of broccoli. However, since in AFD all the experimental factors significantly affected these properties, it should be possible to optimize the operating conditions in AFD in order to maximize the anti-radical power, Se concentration and polyphenols content. Besides, the content of antioxidant compounds, specifically polyphenols and Se, was considerably higher in broccoli subjected to AFD. As a consequence, AFD seems promising in the functional food industry.

\section{Acknowledgments}

The authors wish to thank the financial support of FONDECYT Grants $\mathrm{Nr}$ 1100437 and 1130384.

\section{References}

1. Moreno DA, Carvajal M, López-Berenguer C, García-Viguera C (2006) Chemical and biological characterization of nutraceutical compounds of broccoli. Journal of Pharmaceutical and Biomedical Analysis 41: 1508-1522.

2. Witte KK, Clark AL, Cleland JG (2001) Chronic heart failure and micronutrients. Journal of the American College of Cardiology 37: 1765-1774.

3. Keck AS, Finley JW (2004) Cruciferous vegetables: cancer protective mechanisms of glucosinolate hydrolysis products and selenium. Integr Cancer Ther 3: 5-12

4. D'Archivio M, Filesi C, Di Benedetto R, Gargiulo R, Giovannini C (2007) Polyphenols, dietary sources and bioavailability. Annali dell'Istituto Superiore di Sanità 43: 348-361.
5. Mahn A, Reyes A (2012) An overview of health-promoting compounds of broccoli (Brassica oleracea var italica) and the effect of processing. Food Science and Technology International 18: 503-514.

6. Mahn A, Zamorano M, Barrientos H, Reyes A (2012) Optimization of a process to obtain selenium-enriched freeze-dried broccoli with high antioxidant properties. LWT- Food Science and Technology 47: 267-273.

7. Mahn AV, Antoine P, Reyes A (2011) Optimization of Drying Kinetics and Quality Parameters of Broccoli Florets. International Journal of Food Engineering.

8. Reyes A, Bubnovich V, Bustos R, Vásquez M, Vega R (2010) Comparative study of different process conditions of freeze-drying of 'murtilla' berry. Drying Technology 28: 1416-1425.

9. Kumpulainen J, Raittila AM, Lehto J, Koivistoinen P (1983) Electrothermal atomic absorption spectrometric determination of selenium in foods and diets. Journal of the Association of Official Analytical Chemists 66: 1129-1135.

10. Vinson JA, Xuehui S, Zubik L, Bose $P$ (2001) Phenol antioxidant quantity and quality in foods: Fruits. Journal of Agricultural and Food Chemistry 49: 53155321.

11. Atoui AK, Mansouri A, Boskou G, Kefalas $P$ (2005) Tea and herbal infusions: their antioxidant activity and phenolic profile. Food Chemistry 89: 27-36.

12. Brand Williams W, Cuvelier ME, Berset C (1995) Use of a Free Radical Method to Evaluate Antioxidant Activity. LWT - Food Science and Technology 28: 2530.

13. Box GEP, Hunter WG, Hunter JS (1989) Statistics for experimenters. An introduction to design, data analysis, and model building. Reverté, Spain.

14. Reyes A, Mahn A, Huenulaf $P$ (2011) Drying of apple slices in atmospheric and vacuum freeze-drier. Drying Technology 29: 1076-1089. 